\title{
Theophylline and acetazolamide reduce sleep-disordered breathing at high altitude
}

\author{
R. Fischer*, S.M. Lang\#, M. Leitt*, M. Thiere*, U. Steiner*, R.M. Huber*
}

Theophylline and acetazolamide reduce sleep-disordered breathing at high altitude. $R$. Fischer, S. M. Lang, M. Leitl, M. Thiere, U. Steiner, R. M. Huber. C)ERS Journals Ltd 2004.

ABSTRACT: A randomised, double-blind, placebo-controlled study was conducted to evaluate the effects of theophylline and acetazolamide in the treatment of sleepdisordered breathing (SDB) after fast ascent to high altitude $(3,454 \mathrm{~m})$.

The study was conducted at a high-altitude research laboratory and included 30 healthy male volunteers. Study medication was either oral slow release theophylline $\left(2 \times 250 \mathrm{mg} \cdot \mathrm{day}^{-1}\right)$, oral acetazolamide $\left(2 \times 250 \mathrm{mg} \cdot \mathrm{day}^{-1}\right)$ or a matched placebo tablet. Polysomnographic measurements were performed during two consecutive nights, and acute mountain sickness, pulse rate, oxyhaemoglobin saturation and arterial blood gases were assessed three times a day.

Without active medication, the apnoea/hypopnoea index (AHI) was highly pathological (median $16.2 \cdot \mathrm{h}^{-1}$, range 2-92). Both theophylline and acetazolamide normalised SDB (median AHI $2.5 \cdot h^{-1}$, range $0-11 ; 4.2 \cdot h^{-1}$, range $0-19$, respectively) and reduced oxyhaemoglobin desaturations during sleep (median desaturation index placebo $41.5 \cdot h^{-1}$, range $6-114$; acetazolamide $6.5 \cdot h^{-1}$, range 3-28; theophylline $8.5 \cdot h^{-1}$, range 3-32). Compared with the high amount of central apnoeas or hypopnoeas, the number of obstructive events during sleep was very low in all groups $\left(<4 \cdot h^{-1}\right)$. In contrast to theophylline, acetazolamide significantly improved basal oxyhaemoglobin saturation during sleep (86.2 $\pm 1.7 \%$ versus $81.0 \pm 3.0 \%)$.

The authors conclude that both oral slow release theophylline and acetazolamide are effective to normalise high-altitude sleep-disordered breathing.

Eur Respir J 2004; 23: 47-52.
*Dept of Medicine, Division of Pneumology, Medizinische Klinik - Innenstadt, University of Munich, Munich, and ${ }^{\#}$ Klinik Bad Reichenhall, Bad Reichenhall, Germany.

Correspondence: R. Fischer, Pneumology, Medizinische Klinik - Innenstadt, LMU München, Ziemssenstrasse 1 D-80336 München, Germany.

Fax: 498951604905

E-mail: rainald.fischer@med.uni-muenchen.de

Keywords: Acetazolamide high altitude sleep-disordered breathing theophylline

Received: December 62002

Accepted after revision: June 272003

This study was supported by Deutsche Akademie für Flug- und Reisemedizin, Frankfurt and Radiometer Inc., Munich, Germany.
Sleep-disordered breathing (SDB) of the Cheyne-Stokes type is commonly found during sleep at high altitude [1-3]. This typical breathing pattern with waxing and waning of the tidal volume is associated with profound desaturations and reduced overall oxygen saturation during sleep, but its association with an increased number of arousals is a matter of ongoing debate $[4,5]$. The use of acetazolamide at high altitude is known to reduce the time spent with periodic breathing, to improve overall oxygen saturation during sleep and to reduce the number of arousals [6].

Acetazolamide inhibits the renal enzyme carbonic anhydrase, which leads to metabolic acidosis, and, as a result, increases ventilation and oxygenation. The beneficial effects of this drug at high altitude are thought to be due to this improved oxygenation. Although serious side-effects of acetazolamide are uncommon, an alternative treatment option would be desirable for patients with known sulphonamide intolerance. In a previous, prospective, randomised study, the authors were able to show that theophylline has a positive impact on symptoms of acute mountain sickness (AMS), compared with placebo [7]. As theophylline was shown to be effective in reducing periodic breathing in premature newborns and in patients with severe heart failure $[8,9]$, a similar effect in high-altitude periodic breathing could be assumed.

Therefore, a randomised, double-blind, placebo-controlled study was conducted to evaluate the effect of theophylline and acetazolamide on periodic breathing and arterial oxyhaemoglobin desaturations during sleep at high altitude. The aims were to compare the efficacy of theophylline and acetazolamide in normalising high-altitude SDB and in decreasing nocturnal oxyhaemoglobin desaturation.

\section{Methods}

\section{Study population}

Healthy male volunteers $(n=30)$ gave informed written consent to participate in the study. Of 30 subjects, 22 were engaged in regular mountaineering activities in their spare time, but none of them had experienced altitudes above $3,500 \mathrm{~m}$. The study protocol was approved by the local ethics committee. Exclusion criteria were females, smoking, previous pulmonary disease and concomitant medication. Inclusion criteria were normal weight (body mass index (BMI) $<25$ ) and a medical history of constant good health.

\section{Design of the study}

The double-blind, randomised, placebo-controlled prospective study was performed at the high-alpine research station $(3,454 \mathrm{~m})$ of the University of Berne, Switzerland. The study period (total $46 \mathrm{~h}$ ) included two consecutive nights, and two daytime periods including the evening of the first day $(6 \mathrm{~h})$, 
the whole second day $(18,24,30 \mathrm{~h})$ and the morning of the third day (42 h). Physical exercise (except a fast 1-h walk of all subjects to the Moensjoch hut at $3,750 \mathrm{~m}$ on the first evening) and drugs were not allowed.

\section{Study medication}

Ten subjects were each randomly allocated to $250 \mathrm{mg}$ oral slow-release theophylline (Euphylong; Byk Gulden, Konstanz, Germany), $250 \mathrm{mg}$ acetazolamide (Diamox; Lederle, Muenster, Germany) or placebo (matched tablets) twice daily, starting 3 days before the journey and ending on the morning of the last day. According to experience from a previous study [7], the authors expected the dosage of theophylline to improve sleeping patterns without increased incidence of side-effects.

\section{Study parameters}

Arterialised capillary blood was collected at 6, 18, 24, 30 and $42 \mathrm{~h}$ to measure arterial oxygen tension $\left(\mathrm{Pa}, \mathrm{O}_{2}\right)$, arterial carbon dioxide tension $\left(\mathrm{Pa}, \mathrm{CO}_{2}\right)$ and $\mathrm{pH}(\mathrm{ABL} 5$; Radiometer Inc., Copenhagen, Denmark). Theophylline serum levels were measured in the morning and evening before the intake of the

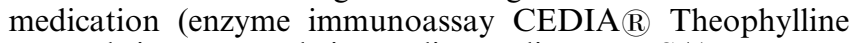
II; Boehringer Mannheim, Indianapolis, IN, USA).

Standard polysomnography was performed with a 32channel polysomnographic device (Sleep Screener; Jaeger Inc., Wuerzburg, Germany) to measure sleep stages (twochannel electroencephalogram (EEG) C4-A1 and C3-A2 according to the international $10 / 20$ system, submental electromyograms (EMGs), electro-oculogram), respiratory function (thoracoabdominal excursions, nasal cannulas, snoring microphone, transcutaneous pulse oximetry), heart rate (two-lead electrocardiogram) and periodic leg movements (tibialis anterior EMGs).

An experienced sleep specialist who was blinded for the administered medication scored the polysomnograms manually. Sleep stages were defined according to the standard criteria of RECHTSCHAFFEN and KALES [10], and arousals were classified following the American Sleep Disorders Association criteria [11]. To avoid arbitrary assignment of respiratory events due to the sometimes difficult interpretation of respiratory signals (measurement of oral/nasal flow by a thermistor is known to underestimate hypoventilations [12]), hypopnoea, apnoea, desaturations, apnoea/hypopnoea index (AHI) and desaturation index (DI) were defined according to standard criteria [13].

An episode of apnoea was diagnosed if complete cessation of inspiratory airflow occurred for $\geqslant 10 \mathrm{~s}$. An episode of obstructive apnoea was defined as the absence of airflow in the presence of rib cage and abdominal movements. An episode of central apnoea was defined as the absence of rib cage and abdominal excursions together with the absence of airflow. Hypopnoea was defined as a $>40 \%$ reduction of airflow lasting $\geqslant 10 \mathrm{~s}$, associated with a $4 \%$ (or more) decrease in arterial oxyhaemoglobin saturation or an EEG arousal, or both. Desaturations were defined as a fall in oxyhaemoglobin saturation of $\geqslant 4 \%$ over a period of $8-60 \mathrm{~s}$, the number of desaturations per hour was referred to as DI. The number of episodes of apnoea and hypopnoea per hour was referred to as AHI.

\section{Data analysis}

The calculated power of the study with a type-I error of $2.5 \%$ (for comparison of placebo versus acetazolamide or theophylline) was $81.2 \%$ with 10 subjects in each group, when a mean difference of $>30$ events $\cdot \mathrm{h}^{-1}\left(\right.$ SD $\left.20 \cdot \mathrm{h}^{-1}\right)$ was assumed. Analysis of variance procedures were performed and the least significant difference post hoc test to compare the three groups for sleep indices, respiratory indices, blood-gas parameters and heart rate. A p-value of $<0.05$ was regarded as statistically significant. Values are expressed as mean \pm SD or as median (range).

\section{Results}

The three study groups did not differ significantly in mean age and BMI (data not shown). Mean theophylline serum levels of the theophylline group are given in table 1 .

\section{Arterial blood gases}

The results of blood-gas measurements are shown in tables 2 and 3. Arterial oxyhaemoglobin tension was significantly higher by increased alveolar ventilation in the acetazolamide group compared with the placebo or theophylline group. In the placebo group, an increase was found in $\mathrm{Pa}, \mathrm{O}_{2}$ and a decrease in $\mathrm{Pa}, \mathrm{CO}_{2}$ during the study period, which

Table 1.-Mean serum theophylline levels in the theophylline group

Variable Theophylline serum levels $\mu \mathrm{g} \cdot \mathrm{mL}^{-1}$

\begin{tabular}{ll}
\hline First evening & $6.12 \pm 1.94$ \\
First morning & $5.25 \pm 1.67$ \\
Second evening & $5.28 \pm 1.17$ \\
Second morning & $4.20 \pm 1.12$
\end{tabular}

Data are presented as mean $\pm \mathrm{SD}$.

Table 2. - Arterial oxygen tension during stay at high altitude

\begin{tabular}{lccc}
\hline $\begin{array}{l}\text { Period at } \\
\text { altitude } h\end{array}$ & $\begin{array}{c}\text { Placebo } \\
\mathrm{kPa}\end{array}$ & $\begin{array}{c}\text { Acetazolamide } \\
\mathrm{kPa}\end{array}$ & $\begin{array}{c}\text { Theophylline } \\
\mathrm{kPa}\end{array}$ \\
\hline 6 & $6.3 \pm 1.0$ & $8.0 \pm 1.1^{*}$ & $6.8 \pm 0.6$ \\
18 & $7.2 \pm 0.5$ & $8.0 \pm 0.5^{* *}$ & $7.3 \pm 0.3$ \\
24 & $7.0 \pm 0.6$ & $8.3 \pm 0.6^{* *}$ & $6.9 \pm 0.4$ \\
30 & $7.0 \pm 0.7$ & $8.1 \pm 1.6^{*}$ & $7.0 \pm 0.5$ \\
42 & $7.5 \pm 1.3$ & $7.9 \pm 0.4^{\#}$ & $7.1 \pm 0.6$ \\
\hline
\end{tabular}

Data are presented as mean \pm SD. *: $\mathrm{p}<0.05$ versus corresponding values in the placebo group and the theophylline group; **: $\mathrm{p}<0.01$ versus corresponding values in the placebo group and the theophylline group; ${ }^{\#}$ : $\mathrm{p}=0.042$ versus corresponding value in the theophylline group.

Table 3. - Arterial carbon dioxide tension during stay at high altitude

\begin{tabular}{lccc}
\hline $\begin{array}{l}\text { Period at } \\
\text { altitude } h\end{array}$ & $\begin{array}{c}\text { Placebo } \\
\mathrm{kPa}\end{array}$ & $\begin{array}{c}\text { Acetazolamide } \\
\mathrm{kPa}\end{array}$ & $\begin{array}{c}\text { Theophylline } \\
\mathrm{kPa}\end{array}$ \\
\hline 6 & $4.7 \pm 0.5$ & $3.7 \pm 0.2^{* *}$ & $4.4 \pm 0.3$ \\
18 & $4.4 \pm 0.3$ & $3.5 \pm 0.2^{* *}$ & $4.3 \pm 0.3$ \\
24 & $4.4 \pm 0.4$ & $3.5 \pm 0.3^{* *}$ & $4.3 \pm 0.2$ \\
30 & $4.3 \pm 0.4$ & $3.5 \pm 0.2^{* *}$ & $4.0 \pm 0.4$ \\
42 & $4.1 \pm 0.3$ & $3.5 \pm 0.2^{* *}$ & $4.2 \pm 0.3$ \\
\hline
\end{tabular}

Data are presented as mean $\pm \mathrm{SD}$. $* *: \mathrm{p}<0.01$ versus corresponding values in the placebo group and the theophylline group. 


\begin{tabular}{|c|c|c|c|}
\hline & Placebo & Acetazolamide & Theophylline \\
\hline \multicolumn{4}{|l|}{ First night } \\
\hline Desaturation index $n \cdot h^{-1}$ & $41.5(6-114)$ & $6.5(3-28)^{* *}$ & $8.5(3-32)^{* *}$ \\
\hline AHI $n \cdot h^{-1}$ & $16.2(3-92)$ & $2.5(0-11)$ & $4.2(0-19)^{* *}$ \\
\hline Basal oxyhaemoglobin saturation $\%$ & $78.9(71-84)$ & $86.8(83-88)^{* *}$ & $80.3(77-86)$ \\
\hline \multicolumn{4}{|l|}{ Second night } \\
\hline Desaturation index $n \cdot h^{-1}$ & $24.0(6-89)$ & $5.0(4-8)^{* *}$ & $8.0(2-31)^{* *}$ \\
\hline AHI second night $n \cdot h^{-1}$ & $6.9(1-77)$ & $1(0-6)^{* *}$ & $3.5(1-6)^{* *}$ \\
\hline Basal oxyhaemoglobin saturation \% & $81.7(67-86)$ & $86.5(83-88)^{\#}$ & $82.5(76-88)$ \\
\hline
\end{tabular}

Data are presented as median (range). AHI: apnoea/hypopnoea index. ${ }^{* *}: \mathrm{p}<0.01$ versus corresponding values in the placebo group; ${ }^{*}: \mathrm{p}<0.05$ versus corresponding values in the placebo group.

was attributed to the ongoing acclimatisation process. Acetazolamide caused lower $\mathrm{pH}$ values, compared with placebo and theophylline $(7.39 \pm 0.02$ versus $7.45 \pm 0.02$ or $7.45 \pm 0.03$, respectively).

\section{Sleep studies: respiratory patterns}

The sleep studies of two subjects (one with theophylline, one with acetazolamide) of the second night were lost due to a technical defect. The mean recording time was $5.6 \mathrm{~h}$ in the first night and $6.0 \mathrm{~h}$ in the second night. There was no significant difference in recording time between the three study groups (table 4).

The exposure to altitude induced SDB predominantly of the Cheyne-Stokes type during the night in nearly all subjects. Central apnoeas or hypopnoeas accounted for $98 \%$ of all respiratory events during sleep in the placebo group, for $71 \%$ in the acetazolamide group and for $78 \%$ in the theophylline group. The other respiratory events were mostly hypopnoeas of the obstructive or mixed type (table 4).

There was a profound difference in the number of events between the placebo group and the treatment groups. In the placebo group, the median AHI $\left(16.2 \cdot \mathrm{h}^{-1}\right.$, range $\left.3-92\right)$ and the median DI $\left(41.5 \cdot \mathrm{h}^{-1}\right.$, range $\left.6-114 \cdot \mathrm{h}^{-1}\right)$ were highly pathological in the first night. A mild reduction in total event numbers was observed in the second night $(21 \%$ in the placebo group versus $39 \%$ in the theophylline group versus $63 \%$ in the acetazolamide group).

In contrast to the severely impaired respiration during the night in the placebo group, both acetazolamide and theophylline reduced periodic breathing substantially and normalised nocturnal breathing patterns. The median AHI was $<5 \cdot \mathrm{h}^{-1}$ in both nights. However, both groups included some individuals with higher AHI, with a maximum score of $11 \cdot \mathrm{h}^{-1}$ in the acetazolamide group and $19 \cdot \mathrm{h}^{-1}$ in the theophylline group. Nevertheless, these maximum values were still below the mean values of the placebo group (table 4).

The relative incidence of obstructive or mixed respiratory events was higher with both drugs, compared with the placebo group. However, the mean absolute number of respiratory events other than central apnoeas or hypopnoeas was not significantly different in all three groups. During the first night, 2.3 events night $^{-1}$ (range $0-10$ ) were found in the placebo group, 4.5 events night $^{-1}$ (range 0-16) in the acetezolamide group and 7.1 events $\cdot$ night $^{-1}$ (range $\left.0-24\right)$ in the theophylline group $(\mathrm{p}>0.1)$.

The discrepancy between the higher DI and the lower AHI in all groups is due to the frequent Cheyne-Stokes breathing pattern. This waxing and waning breathing pattern induces periodic desaturations. However, these respiratory events do often not meet the standard criteria defined in the method section for apnoea or hypopnoea, generally due to the short duration $(<10 \mathrm{~s})$ of the event or to insufficient decrease of the thermistor signal (figs 1 and 2).

In accordance with daytime changes of arterial blood gases, basal and nocturnal oxyhaemoglobin saturation differed significantly between acetazolamide (improvement $>5 \%$ ) and theophylline and placebo, respectively, during the night (no change).

\section{Sleep studies: sleep stages and arousals}

When analysing sleep quality during both nights, it is striking that overall sleep architecture (including substantial rapid eye movement (REM) sleep and slow-wave non-REM sleep) was very well preserved. A distinct "first night effect" was not found as the sleep efficiency remained virtually unchanged during both nights. The relative amount of sleep stages corresponded to normal values found in healthy volunteers in lowland studies. The only significant difference found between the three groups was a slightly reduced amount of stage-I sleep in the acetazolamide group in the first night, in favour of an increase of stage IV and REM sleep. In spite of the high AHI, the number of arousals in the treatment group relative to the placebo group only achieved significance in the second night at high altitude (table 5).

\section{Side-effects}

All the subjects were naive to the drugs employed in this study. In addition to the symptoms related to AMS, no major side-effects of the drugs were reported. Of the subjects on acetazolamide, $60 \%$ observed paresthesias in hands and feet and impaired taste of fizzy drinks. Of the subjects on theophylline, $70 \%$ reported heart palpitations. Nearly all subjects (including placebo) reported increased diuresis, especially during the night.

\section{Discussion}

These data demonstrate that nocturnal oxygen desaturations and altered sleep respiratory patterns, reflected in a pathological AHI and DI, occurred with high frequency in healthy volunteers acutely exposed to an altitude of 3,454 m, and that the short-term administration of theophylline or acetazolamide virtually normalised SDB and reduced associated oxyhaemoglobin desaturations.

In spite of different mechanisms of action of both drugs, no significant difference was found regarding the efficacy of both drugs to reduce sleep disturbance at high altitude. Both drugs reduced SDB to a very low level. 


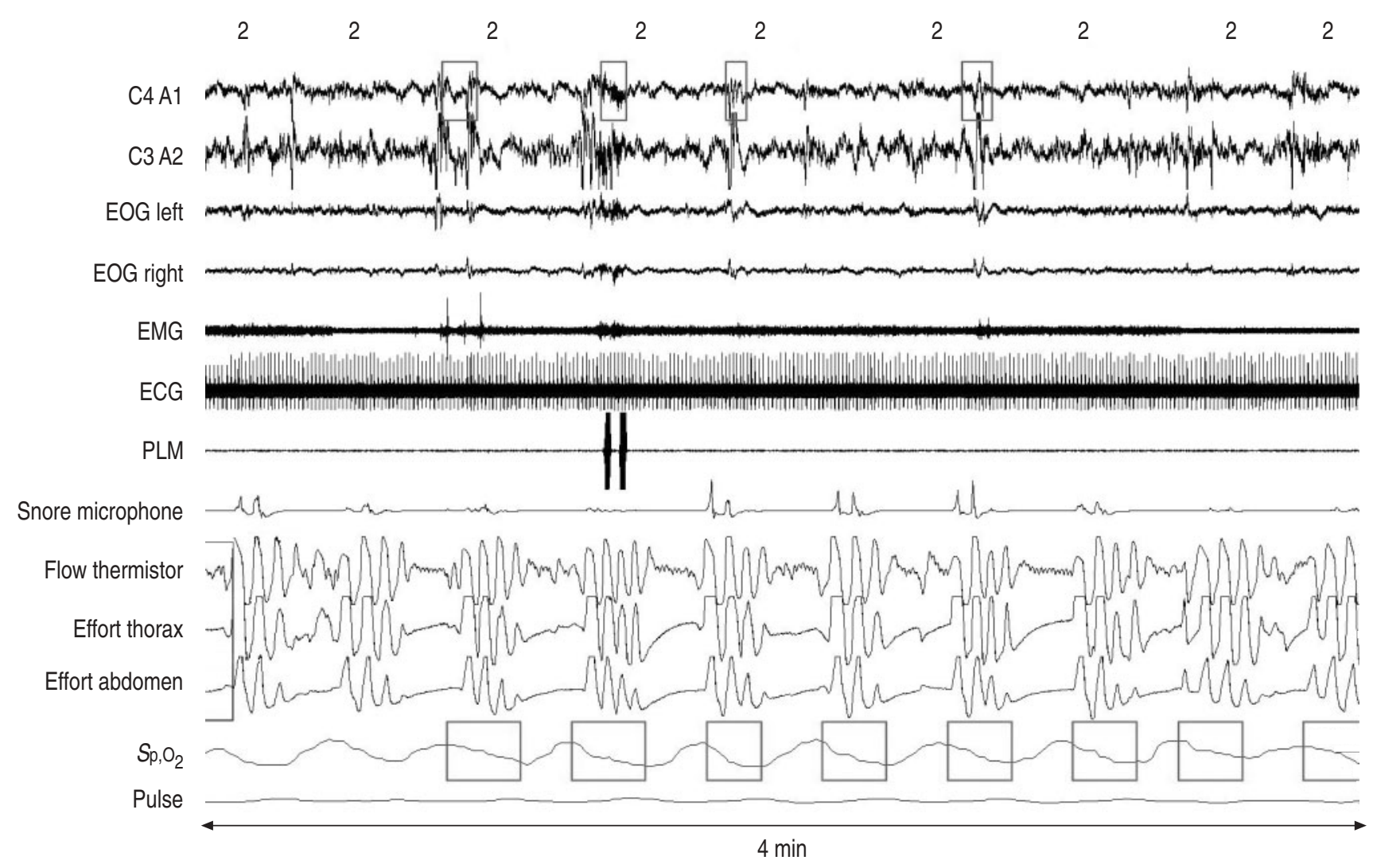

Fig. 1.- Typical episode of periodic breathing with central apnoeas or hypopnoeas during stage-II sleep, desaturations of $>4 \%$ (boxes in the arterial oxygen saturation measured by pulse oximetry $\left(\mathrm{S}_{\mathrm{p}, \mathrm{O}_{2}}\right)$ line) and several arousals (boxes in the C4-A1 line). The following channels are shown (top to bottom): electroencephalogram (EEG) C4-A1, EEG C3-A2, electro-oculogram (EOG) right eye, EOG left eye, electromyogram (EMG) chin, electrocardiogram (ECG), EMG right leg (periodic leg movements; PLM), snore microphone, oral/nasal flow, rib cage (thorax) effort, abdominal effort, $S_{\mathrm{p}, \mathrm{O}_{2}}$ (upper limit $100 \%$, lower limit $70 \%$ ), pulse rate. Period length 4 min.

The two drugs investigated differ in their proposed mechanisms of action. Acetazolamide increases ventilation by inducing metabolic acidosis through inhibition of the renal carbonic anhydrase with an ensuing increase in ventilation, which is reflected by higher $\mathrm{Pa}, \mathrm{O}_{2}$ and lower $\mathrm{Pa}, \mathrm{CO}_{2}$ values. Therefore, the observed effect of acetazolamide can be attributed to the improved oxygenation caused by hyperventilation and the left shift of the hypercapnic ventilatory response [14]. According to the control theory by KHOO et al. [15], this makes the development of apnoeas after a transient rise of ventilation, e.g. after an arousal, less likely to be due to the decreased oscillations of the corrective breathing actions (hyperventilation or apnoea).

By contrast, theophylline was previously shown to increase ventilation and carbon dioxide production without affecting the slope of the hypercapnic ventilatory response [16]. An increase in tidal volume (without change in respiratory frequency) was thought to be due to the competition with adenosine at some of its central receptor sites [17]. In the present investigation, it seems likely that theophylline activated central respiratory receptors and reversed the hypoxic ventilatory depression [18]. This resulted in a more in-phase response of ventilation to altered blood gases with consecutive reduction of periodic breathing [19, 20]. The fact that the effects of theophylline observed in this investigation occurred at relatively low serum concentrations, suggests that only little activation of central chemoreceptors is necessary to stabilise the out-of-phase breathing oscillations. This finding is important when theophylline is used in a high-altitude setting, as higher serum levels could result in impaired sleep quality in susceptible individuals.

Although both drugs normalised disturbed breathing patterns, no substantial differences in stages of sleep, arousal indices and sleep efficiency were found. However, in contrast to some previous studies, sleep quality was barely impaired even in the control group [3, 21, 22]. The lack of correlation between arousal indices and AHI in this study indicates that the number of arousals was a result of the altitude exposure itself and not secondary to respiratory events [23]. In the second night, there was a trend to lower arousal indices with both active treatments. This is in accordance with the study of WEIL et al. [5], which showed that acetazolamide reduces arousals during high-altitude sleep. As the lowest arousal index was found in the acetazolamide group, this effect may result from increased basal oxygen saturation rather than from reduced periodic breathing.

This study, therefore, provides some evidence that theophylline is effective to reduce high-altitude, disturbed breathing patterns in susceptible subjects. Theophylline could be useful in patients with known sulphonamide intolerance and in those patient groups where the additional properties of this drug, such as antiobstructive effects as well as improved diaphragmal contractility preventing failure of respiratory muscles [24], are desirable. These patient groups include individuals with chronic obstructive pulmonary disease when travelling and staying at higher altitudes.

Although acetazolamide remains the standard treatment of acute mountain sickness and periodic breathing at high 


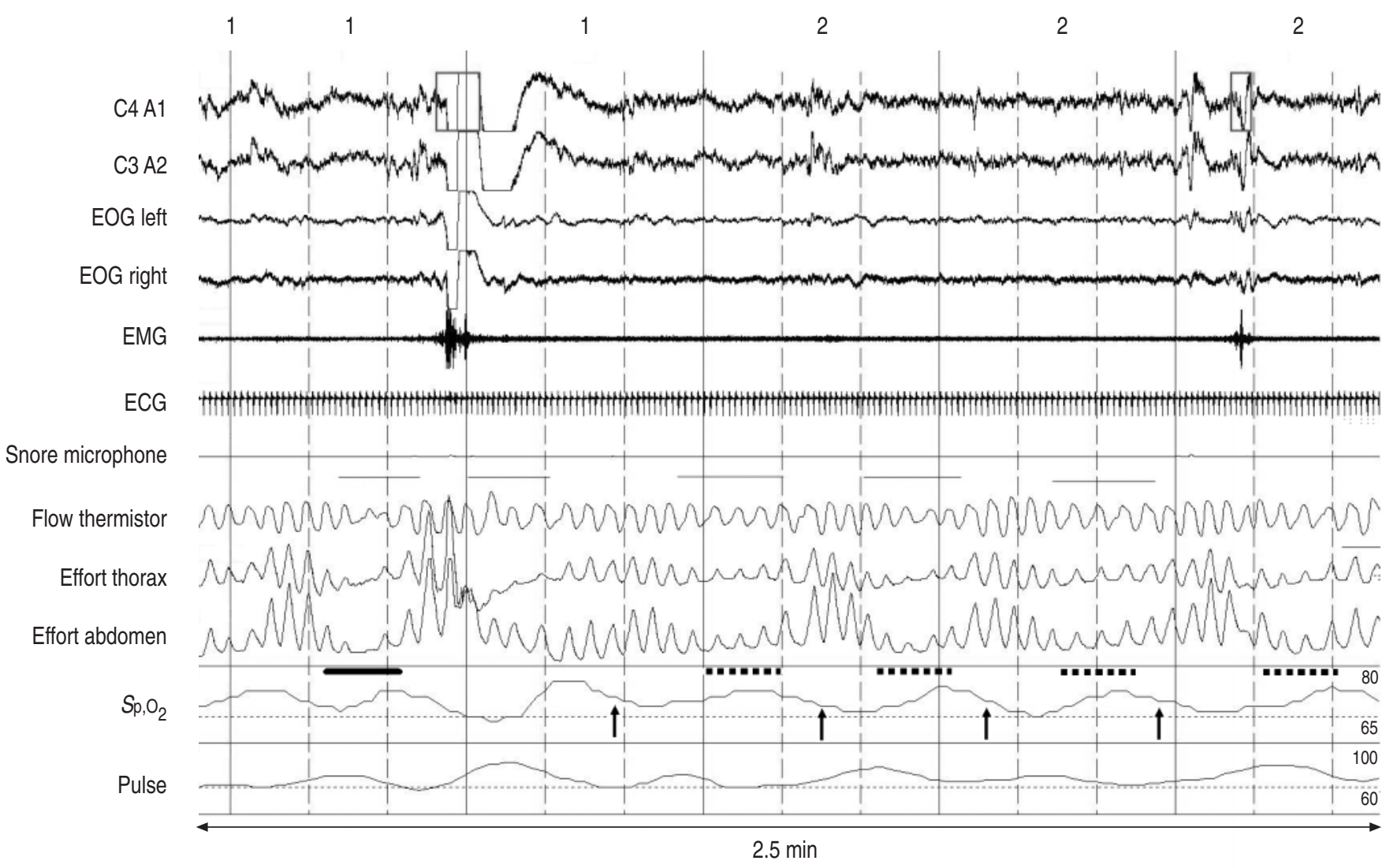

Fig. 2. - Breathing irregularity with a central hypopnoea at the beginning (bold line) and arousal. The following desaturations (arrows) are not preceeded by respiratory events meeting the criteria defined in the methods sections, although periodic breathing is detectable in the rib cage (thorax) and abdominal effort signals (dotted lines). Therefore, the number of respiratory events is lower than the number of desaturations in this study. Period length $2.5 \mathrm{~min}$. Channel order is identical to figure 1. EOG: electro-oculogram; EMG: electromyogram; ECG: electrocardiogram; $S_{\mathrm{p}, \mathrm{O}_{2}}$ : arterial oxygen saturation measured by pulse oximetry.

Table 5. - Sleep stage parameters during stay at high altitude

Placebo Acetazolamide Theophylline

First night

$\begin{array}{lccc}\text { Sleep efficiency \% } & 92.7 \pm 2.1 & 81.0 \pm 4.1 & 86.7 \pm 8.4 \\ \text { Stage REM \% } & 11.1 \pm 5.0 & 14.8 \pm 5.4 & 12.7 \pm 5.4 \\ \text { Stage I \% } & 13.8 \pm 7.3 & 5.6 \pm 2.6^{\#} & 11.3 \pm 6.9 \\ \text { Stage II \% } & 54.1 \pm 7.7 & 54.3 \pm 5.5 & 51.5 \pm 11.4 \\ \text { Stage III \% } & 6.3 \pm 2.4 & 6.8 \pm 3.5 & 7.7 \pm 2.6 \\ \text { Stage IV \% } & 12.7 \pm 8.5 & 16.6 \pm 5.1 & 11.2 \pm 5.2 \\ \text { Arousal index } \mathrm{n} \cdot \mathrm{h}^{-1} & 20.2 \pm 20.5 & 12.6 \pm 7.4 & 12.5 \pm 6.4 \\ \text { Second night }^{\oplus} & & & \\ \text { Arousal index } \mathrm{n} \cdot \mathrm{h}^{-1} & 20.3 \pm 15.0 & 10.3 \pm 4.0^{+} & 13.6 \pm 6.8\end{array}$

Data are given as mean \pm SD. REM: rapid eye movement; ${ }^{\top}$ only significant differences are shown; ${ }^{\#}: \mathrm{p}=0.04$ versus corresponding values in the placebo group; ${ }^{+}: \mathrm{p}=0.028$ versus corresponding values in the placebo group.

altitude, the authors conclude that theophylline is an effective alternative with possible benefits for patients with concomitant respiratory disorders (e.g. exercise or cold-induced bronchospasm) or renal disease.

\section{References}

1. Anholm JD, Powles AC, Downey R III, et al. Operation Everest II: arterial oxygen saturation and sleep at extreme simulated altitude. Am Rev Respir Dis 1992; 145: 817-826.
2. Salvaggio A, Insalaco G, Marrone O, et al. Effects of highaltitude periodic breathing on sleep and arterial oxyhaemoglobin saturation. Eur Respir J 1998; 12: 408-413.

3. Zielinski J, Koziej M, Mankowski M, et al. The quality of sleep and periodic breathing in healthy subjects at an altitude of 3,200 m. High Alt Med Biol 2000; 1: 331-336.

4. Khoo MC, Anholm JD, Ko SW, et al. Dynamics of periodic breathing and arousal during sleep at extreme altitude. Respir Physiol 1996; 103: 33-43.

5. Weil JV, Kryger MH, Scoggin CH. Sleep and breathing at high altitude. In: Guilleminault C, Dement W, eds. Sleep Apnea Syndromes Proceedings. New York, Liss, 2002; pp. 119-136.

6. Hackett PH, Roach RC, Harrison GL, Schoene RB, Mills WJ Jr. Respiratory stimulants and sleep periodic breathing at high altitude. Almitrine versus acetazolamide. Am Rev Respir Dis 1987; 135: 896-898.

7. Fischer R, Lang SM, Steiner U, et al. Theophylline improves acute mountain sickness. Eur Respir J 2000; 15: 123-127.

8. Cordoba E, Gerhardt T, Rojas M, Duara S, Bancalari E. Comparison of the effects of acetazolamide and aminophylline on apnea incidence and on ventilatory response to $\mathrm{CO}_{2}$ in preterm infants. Pediatr Pulmonol 1994; 17: 291-295.

9. Javaheri S, Parker TJ, Wexler L, Liming JD, Lindower P, Roselle GA. Effect of theophylline on sleep-disordered breathing in heart failure. $N$ Engl J Med 1996; 335: 562567.

10. Rechtschaffen A, Kales A. A manual of standardized terminology, techniques and scoring system for sleep stages of human subjects. Washington, US Government Printing Office, 1968. 
11. American Sleep Disorders Association. EEG arousals: scoring rules and examples: a preliminary report from the Sleep Disorders Atlas Task Force of the American Sleep Disorders Association. Sleep 1992; 15: 173-184.

12. Series F, Marc I. Nasal pressure recording in the diagnosis of sleep apnoea hypopnoea syndrome. Thorax 1999; 54: 506510.

13. American Academy of Sleep Medicine Task Force. Sleeprelated breathing disorders in adults: recommendations for syndrome definition and measurement techniques in clinical research. The Report of an American Academy of Sleep Medicine Task Force. Sleep 1999; 22: 667-689.

14. White DP, Zwillich CW, Pickett CK, Douglas NJ, Findley LJ, Weil JV. Central sleep apnea. Improvement with acetazolamide therapy. Arch Intern Med 1982; 142: 1816-1819.

15. Khoo MC, Kronauer RE, Strohl KP, Slutsky AS. Factors inducing periodic breathing in humans: a general model. J Appl Physiol 1982; 53: 644-659.

16. Javaheri S, Guerra L. Lung function, hypoxic and hypercapnic ventilatory responses, and respiratory muscle strength in normal subjects taking oral theophylline. Thorax 1990; 45 : 743-747.

17. Darnall RA Jr. Aminophylline reduces hypoxic ventilatory depression: possible role of adenosine. Pediatr Res 1985; 19: 706-710.

18. Javaheri S, Teppema LJ, Evers JA. Effects of aminophylline on hypoxemia-induced ventilatory depression in the cat. J Appl Physiol 1988; 64: 1837-1843.

19. Khoo MC, Gottschalk A, Pack AI. Sleep-induced periodic breathing and apnea: a theoretical study. J Appl Physiol 1991; 70: 2014-2024.

20. Xie A, Skatrud JB, Dempsey JA. Effect of hypoxia on the hypopnoeic and apnoeic threshold for $\mathrm{CO}(2)$ in sleeping humans. J Physiol 2001; 535: 269-278.

21. Eichenberger U, Weiss E, Riemann D, Oelz O, Bartsch P. Nocturnal periodic breathing and the development of acute high altitude illness. Am J Respir Crit Care Med 1996; 154: $1748-1754$.

22. Goldenberg F, Richalet JP, Onnen I, Antezana AM. Sleep apneas and high altitude newcomers. Int J Sports Med 1992; 13: Suppl. 1; S34-S36.

23. Reite M, Jackson D, Cahoon RL, Weil JV. Sleep physiology at high altitude. Electroencephalogr Clin Neurophysiol 1975; 38: 463-471.

24. Marchand E, Decramer M. Respiratory muscle function and drive in chronic obstructive pulmonary disease. Clin Chest Med 2000; 21: 679-692. 